\title{
Signal intensity on MRI of basal ganglia In multiple sclerosis
}

\author{
J Grimaud, J Millar, J W Thorpe, I F Moseley, W I McDonald, D H Miller
}

\begin{abstract}
It has been reported that a relative reduction in signal intensity on $T 2$ weighted MRI may be seen in the basal ganglia of patients with multiple sclerosis and furthermore that this is due to excessive iron deposition. The basal ganglia are, however, rarely involved clinically or pathologically in multiple sclerosis, casting some doubt on this finding. Therefore MRI was carried out in 46 patients with definite multiple sclerosis and 42 age matched controls. Contiguous, $5 \mathrm{~mm}$ thick axial dual echo spin-echo images of the brain were obtained on a $1.5 \mathrm{~T}$ imager. Visual rating scales were used to measure the lesion load as well as the signal intensity of the globus pallidus, putamen, caudate nucleus, substantia nigra, red nucleus, and thalamus. There was a mild degree of low signal intensity in the patient group in the thalamus only. The signal intensity of the thalamus and putamen was never lower than that of the globus pallidus. Low signal in the basal ganglia is rarely, if ever, found in multiple sclerosis and is not a useful radiological sign.
\end{abstract}

(F Neurol Neurolosurg Psychiatry 1995;59:306-308)

Keywords: multiple sclerosis; magnetic resonance imaging; basal ganglia; iron

Increased deposition of iron in the brain has been found adjacent to chronic multiple sclerosis plaques. ${ }^{1}$ It has been reported that a relative reduction in signal intensity on $\mathrm{T} 2$ weighted MRI may be seen in the basal ganglia of patients with multiple sclerosis and this has also been attributed to excessive iron deposition. ${ }^{2}$ The low frequency of pathological, MRI, or clinical ${ }^{3}$ involvement of the basal ganglia in multiple sclerosis casts doubt on the validity of this finding. We have therefore studied basal ganglia signal intensity in patients with multiple sclerosis and controls.

\section{Method}

We studied 46 patients with definite multiple sclerosis according to the Poser criteria ${ }^{4}$ (29 relapsing and remitting, 15 secondary pro- gressive, two primary progressive) and 42 age and sex matched controls: healthy subjects and patients undergoing inveștigation for headache, vertigo, or middle ear disorders in whom multiple sclerosis was excluded clinically. These two groups were defined as follows: sex ratio $(M / F)$ in patients with multiple sclerosis 0.77 and controls 0.68 , mean (SD, range) age of patients with multiple sclerosis $39.9(11.1,19-64)$ years and controls $43.3(11.8,24-72)$ years. Brain MRI was performed with a $1.5 \mathrm{~T}$ imager. Contiguous $5 \mathrm{~mm}$ thick interleaved axial dual echo spin-echo images were obtained with repetition times (TR) of 2000 or $2700 \mathrm{~ms}$, echo times (TEs) of 32 and $80 \mathrm{~ms}$, and one excitation. The field of view was $24 \mathrm{~cm}$, the matrix $192 \times 256$

Signal characteristics of the basal ganglia on long TE images were first analysed in several regions: caudate nucleus, putamen, globus pallidus, dentate and red nuclei, substantia nigra, and thalamus. Signal change within the basal ganglia was rated according to the method proposed by Drayer et $a l^{2}{ }^{2}$ which involves comparing the signal characteristics of the basal ganglia relative to the globus pallidus. Three different patterns are described.

Normal-decreased signal intensity in the globus pallidus, more prominent than in the putamen and much more prominent than in the thalamus.

Equivocal-decreased signal intensity in the globus pallidus, slightly more prominent than in the putamen and more prominent than in the thalamus.

Abnormal-decreased signal intensity in the globus pallidus equal to or less prominent than that in the putamen and caudate and only minimally more prominent or equally prominent to that in the thalamus.

To assess the visual perception of the degree of basal ganglia hypointensity as an absolute rather than one nucleus relative to another a different method was also used. Each of the basal ganglia was scored separately on an arbitrary scale with cortical grey matter as the internal reference ( $0=$ isointense, 3 = maximum low signal intensity).

The size and number of white matter lesions were subsequently scored, with both short and long TE images. All discrete white matter lesions were registered and weighted 
according to their largest diameter. Confluent periventricular lesions, however, were weighted according to their smallest diameter. This allowed the computation of a semiquantitative lesion score as follows: lesion smaller than $5 \mathrm{~mm}$ in diameter $=1$, lesions 5-10 $\mathrm{mm}=2$, larger than $10 \mathrm{~mm}=3$, and confluence added an extra point to the score. We scored a lesion on every slice that it appeared. Patients were separated into four groups on the basis of the degree of MRI abnormality: normal, mild ( $1 \leqslant$ lesion load $<50$ ), moderate $(50 \leqslant$ lesion load $<100)$, severe (lesion load $\geqslant 100$ ).

The relation between the number and severity (normal, mild, moderate, severe) of white matter lesions and basal ganglia signal intensity ratings $(0,1,2$, or 3$)$ on dual echo spin echo images were evaluated with Spearman's rank correlation. The non-parametric Mann-Whitney test was used to compare multiple sclerosis and control groups with respect to the distribution of signal intensity in the basal ganglia.

\section{Results}

Among the 42 control subjects there were no abnormalities in 24 and patchy white matter lesions in 18 (lesion load: mean (SD, range, median) $7.6(9.0,1-32,4)$. Those without abnormal white matter were significantly younger than those with $(40 \cdot 1(10 \cdot 5) v 47.4$ $(12.2) ; P=0.05)$ and lesion load correlated positively with increasing age $(r=0.38$; $P=0.02)$. Scans of all patients with multiple sclerosis showed white matter lesions (lesion load: mean (SD, range, median) 100.9 $(106.6,5-696,78)$. The mean (SD) lesion load in relapsing remitting patients with multiple sclerosis was $100.0(139.0)$ compared with $107.5(54.3)$ in patients with secondary progressive multiple sclerosis (NS). There was no association between lesion load, patient age, or duration of the disease.

With our signal intensity rating scale, the mean basal ganglia low signal intensity in controls varied between 0.00 (for the caudate nucleus) and 1.79 (for the globus pallidus). The thalamic, putaminal, and caudate nuclei had intensities similar to the cortex (median $=0$ ). The globus pallidus and substantia nigra had the lowest intensities compared with the cortex (median $=2$ ). The red nuclei and the dentate nuclei were of intermediate intensity (median $=1$; table).

Signal intensity rating

\begin{tabular}{llllllc}
\hline & \multicolumn{2}{l}{ Patients $(n=46)$} & & & \multicolumn{2}{l}{ Controls $(n=42)$} \\
\cline { 2 - 3 } Nuclei & mean $(S D)$ & median & & mean $(S D)$ & median & Pvalue \\
\hline Globus pallidus & $1.85(0.54)$ & 2 & & $1.79(0.56)$ & 2 & 0.60 \\
Substantia nigra & $1.74(0.65)$ & 2 & & $1.69(0.68)$ & 2 & 0.68 \\
Red nucleus & $1.61(0.65)$ & 2 & & $1.38(0.58)$ & 1 & 0.08 \\
Dentate nucleus & $1.04(0.59)$ & 1 & & $0.83(0.54)$ & 1 & 0.10 \\
Thalamus & $0.56(0.54)$ & 1 & & $0.12(0.33)$ & 0 & $<0.001$ \\
Putamen & $0.28(0.45)$ & 0 & & $0.17(0.38)$ & 0 & 0.20 \\
Caudate & $0.02(0.15)$ & 0 & & $0.00(0.00)$ & 0 & 1 \\
\hline
\end{tabular}

Each of the basal ganglia was scored separately on an arbitrary visual rating scale with cortex as the internal reference $(0=$ isointense, $3=$ maximum hypointensity).
A subtle decrease in signal intensity rating was found in the thalamic nuclei of the patients with multiple sclerosis (mean (SD, median) $0.56(0.54,1)$ compared with the controls $0.12(0.33,0 ; \mathrm{P}<0.001)$. For the other basal ganglia and in particular the putamen, there was no difference between patients with multiple sclerosis and controls. Decreased signal intensity in the basal ganglia did not correlate with age or duration of disease. Similarly, there was no linear correlation between the lesion load in the white matter and the signal intensity of any of the basal ganglia. Greatly reduced signal intensity in the putamen or caudate relative to the globus pallidus as found by Drayer et $a l^{2}$ was not found in any of our patients.

\section{Discussion}

With the advent of high field strength imaging, with long repetition and echo times (T2 weighted), a close correlation has been found between areas of prominent low intensity in the extrapyramidal system and the known sites of preferential accumulation of ferric iron as documented by Perl's stain and other necropsy studies. ${ }^{25-7}$ These findings led to the hypothesis that the low signal intensities (indicating a short $\mathrm{T} 2$ relaxation time) were a direct paramagnetic effect of the high iron concentration, although the exact relation between T2 values and iron concentration remains somewhat controversial. ${ }^{8-10}$

Several independent investigators have suggested that abnormal patterns of iron in the brain, shown either postmortem or inferred from areas of low signal intensity on long TR, long TE MRI are a feature of several neurological disorders including Huntington's chorea, Parkinson's disease, and multisystem atrophy. A consistent decrease in signal intensity on images dependent on T2 in the globus pallidus, substantia nigra, red nucleus, dentate nucleus, and to a lesser extent, the thalamus, putamen, and caudate, is, however, a striking feature of high field strength MRI even in healthy adults. ${ }^{11}$ The normally lower signal intensity in the globus pallidus than in the putamen ${ }^{12}$ was confirmed in the present study $(P<0.0001)$. Interestingly, the results of independent quantitative studies that showed a preferential accumulation of non-haem iron in several basal ganglia, are congruent with our qualitative assessment of signal intensity. In descending order, these studies have found the following iron concentrations: globus pallidus $(21.30 \mathrm{mg}$ iron/100 $\mathrm{g}$ fresh weight), red nucleus $(19.48 \mathrm{mg} / 100 \mathrm{~g})$, substantia nigra $(18.46 \mathrm{mg} / 100 \mathrm{~g})$, putamen $(13.32$ $\mathrm{mg} / 100 \mathrm{~g}$ ), dentate nucleus $(10.35 \mathrm{mg} / 100 \mathrm{~g})$, caudate nucleus $(9.28 \mathrm{mg} / 100 \mathrm{~g})$, and thalamus $(4 \cdot 76 \mathrm{mg} / 100 \mathrm{~g}) .^{713}$

Drayer et $a l^{2}$ although finding no difference in measured T2 relaxation times in the globus pallidus, putamen, and thalamus between normal subjects and patients with MS, did report a highly significant, subjective visual impression of loss of signal intensity in 
the putamen and thalamus relative to the normal low signal intensity of the globus pallidus, which correlated with lesion load in the cerebral white matter. There was a smaller but none the less significant correlation between the measured T2 ratio of the thalamus to globus pallidus in these patients (the putamen to globus pallidus ratio just failed to reach significance). In view of the minimal changes in measured T2 relaxation, the striking degree of hypointensity on subjective analyses was surprising. In our study, there was evidence of only a slight decrease in signal intensity in the thalamus of the multiple sclerosis group. The low signal intensity score in patients with multiple sclerosis was 0 $(n=21)$ or $1(n=24)$ in all but one patient who scored 2; of the controls 37 scored 0 , and five scored 1 . Such slight changes probably would not be apparent on routine MRI studies. In all other basal ganglia, there was no difference between patients with multiple sclerosis and controls with respect to the frequency of the degree of low signal intensity.

It is not clear why iron normally accumulates in the basal ganglia. It is known that iron is resorbed by capillary endothelial cells in the thalamus and other basal ganglia after transferrin binds to a specific receptor on the cell surface. ${ }^{14}{ }^{15}$ It is then transported along axons to their sites of projection where the iron is subsequently released and stored in oligodendrocytes. ${ }^{14}$ There can be considerable loss of axons in multiple sclerosis and this could conceivably cause increased amounts of iron at the site of uptake in the basal ganglia. ${ }^{1516} \mathrm{It}$ is possible that axonal loss in the thalamus is greater than in other structures in the basal ganglia as it is a relay centre for sensory afferent pathways that are very often involved, clinically and pathologically, in multiple sclerosis.

In conclusion, basal ganglia signal hypointensity on long TR/TE images, beyond those seen in normal people, occurs rarely, if ever, in patients with multiple sclerosis.
All MRIs were performed on a scanner provided by the "Multiple Sclerosis Society of Great Britain and Northern Ireland". JG is grateful to the "Association pour la Recherche Ireland". JG is grateful to the "Association pour la Recherche
sur la Sclerose en Plaques" (ARSEP) and the Lilly Industries sur la Sclerose en Plaques" (ARSEP) and the Lilly Industries UK for personal support. We also
contributed so much of their time.

1 Craelius W, Migdal MW, Luessenhop CP, Luessenhop CP, Sugar A, Mihalakis I. Iron deposits surrounding multiple sclerosis plaques. Arch Pathol Lab Med 1982; 106:397-9

2 Drayer B, Burger P, Hurwitz B, Dawson D, Cain J. Reduced signal intensity on MR images of thalamus and putamen in multiple sclerosis : increased iron content? AfNR Am $\mathcal{f}$ Neuroradiol 1987;8:413-9.

3 McAlpine D, Lumsden CE, Acheson ED. Multiple sclerosis: a reappraisal. Baltimore: Williams and Wilkins, 1972:177-8.

4 Poser CM, Paty DW, Scheinberg L, McDonald WI, Davis FA, Ebers GC, et al. New diagnostic criteria for multiple sclerosis: guidelines for research protocols. Ann Neurol 1983;13:227-31.

5 Spatz H. Über den Fe-Nachweis im Gehirn, besonders in Zentren des extrapyramidal-motorischen Systems, I Teil. Z Ges Neurol Psychiat 1922;77:261-390.

6 Spatz $\mathrm{H}$. Über Stoffwechseleigentümlichkeiten in den Stammganglien. $Z$ Ges Neurol Psychiat 1922;78:641-8.

7 Hallgren B, Sourander P. The effect of age on the non-haemin iron in the human brain. $f$ Neurochem 1958;3:41-51.

8 Chen JC, Hardy PA, Clauberg M, Joshi JG, Parravano J, Deck JHN, et al. T2 values in the human brain: comparison with quantitative assays of iron and ferritin. Radiology 1989;173:521-6.

9 Brooks DJ, Luthert P, Gadian D, Marsden CD. Does signal-attenuation on high-field T2- weighted MRI of the brain reflect regional cerebral iron deposition? Observations on the relationship between regional Observations on the relationship between regional
cerebral water proton T2 values and iron levels. $\mathcal{f}$ Neurol Neurosurg Psychiatry 1989;52:108-11.

10 Bizzi A, Brooks RA, Brunetti A, Hill JM, Alger JR, Miletich RS, et al. Role of iron and ferritin in MR imaging of the brain: a study in primates at different field strengths. Radiology 1990;177:59-65.

11 Milton WJ, Atlas SW, Lexas FJ, Mozeley PD, Gur RE. Deep gray matter hypointensity patterns with aging in healthy adults: MR imaging at 1.5 T. Radiology 1991; 181:715-9.

12 Darwin RH, Drayer BP, Riederer SJ, Wang HZ, MacFall JR. T2 estimates in healthy and diseased brain tissue: a comparison using various MR pulse sequences. Radiology 1986,160:375-81.

13 Hoeck A, Demmel U, Schicha H, et al. Trace element concentration in human brain. Brain 1975;98:49-64

14 Hill JM, Ruff MR, Weber RJ, Pert CB. Transferrin receptors in rat brain: neuropeptide-like pattern and relationship to iron distribution. Proc Natl Acad Sci USA 1985; 82:4553-7

15 Dietrich RB, Bradley WG Jr. Iron accumulation in the basal ganglia following severe ischemic-anoxic insults in children. Radiology 1988;168:203-6.

16 Bradley WG. MRI of hemorrhage and iron in the brain. In: Stark DD, Bradley WG, eds. Magnetic resonance imaging. St Louis: Mosby, 1988:359-74. 